\title{
Changes in bioimpedance analysis components before and after hemodialysis
}

\author{
Hyunsuk Kim ${ }^{1}$, Gwang Ho $\mathrm{Choi}^{1}$, Kwang Eon Shim ${ }^{1}$, Jung Hoon Lee ${ }^{1}$, Nam Ju Heo ${ }^{2}$, Kwon-Wook Joo ${ }^{3}$, \\ Jong-Woo Yoon ${ }^{1}$, Yun Kyu $\mathrm{Oh}^{4}$ \\ 'Division of Nephrology, Department of Internal Medicine, Hallym University Chuncheon Sacred Heart Hospital, Hallym University \\ College of Medicine, Chuncheon, Korea \\ ${ }^{2}$ Department of Internal Medicine, Seoul National University Hospital Healthcare System Gangnam Center, Seoul, Korea \\ ${ }^{3}$ Department of Internal Medicine, Seoul National University Hospital, Seoul, Korea \\ ${ }^{4}$ Department of Internal Medicine, Seoul National University Boramae Medical Center, Seoul, Korea
}

\begin{abstract}
Background: This study compared nutritional parameters in hemodialysis (HD) subjects and controls using bioimpedance analysis (BIA) and investigated how BIA components changed before and after HD.

Methods: This cross-sectional study included 147 subjects on maintenance HD from two hospitals and 298 propensity score-matched controls from one healthcare center. BIA was performed pre- and post-HD at mid-week dialysis sessions.

Results: Extracellular water/total body water (ECW/TBW) and waist-hip ratio were higher in the HD patients; the other variables were higher in the control group. The cardiothoracic ratio correlated best with overhydration $(r=0.425$, $P<0.01$ ) in HD subjects. Blood pressure, hemoglobin, creatinine, and uric acid positively correlated with the lean tissue index in controls; however, most of these nutritional markers did not show significant correlations in HD subjects. Normal hydrated weight was predicted to be higher in the pre-HD than post-HD measurements. Predicted ultrafiltration (UF) volume difference based on pre- and post-HD ECW/TBW and measured UF volume difference showed a close correlation $\left(r^{2}=0.924, P<0.01\right)$. Remarkably, the leg phase angle increased in the post-HD period. Conclusion: The estimated normal hydrated weight using ECW/TBW can be a good marker for determining dry weight. HD subjects had higher ECW/TBW but most nutritional indices were inferior to those of controls. It was possible to predict UF volume differences using BIA, but the post-HD increase in leg phase angle, a nutritional marker, must be interpreted with caution.
\end{abstract}

Keywords: Bioimpedance, Hemodialysis, Lean tissue index, Nutritional status, Overhydration

\footnotetext{
Received April 23, 2018; Revised August 14, 2018; Accepted August 21, 2018

Correspondence: Yun Kyu Oh

Department of Internal Medicine, Seoul National University Boramae Medical Center, 20 Boramae-ro 5-gil, Dongjak-gu, Seoul o7061, Korea. E-mail: yoonkyu@snu.ac.kr

ORCID: https://orcid.org/0000-0001-8632-5743

A part of this work was presented at KSN (Korean Society of Nephrology) 2016 in Seoul, South Korea.

Copyright (C) 2018 by The Korean Society of Nephrology

(a) This is an open-access article distributed under the terms of the Creative Commons Attribution Non-Commercial License (http://creativecommons.org/licenses/by-nc-nd/4.0/), which permits unrestricted non-commercial use, distribution, and reproduction in any medium, provided the original work is properly cited.
} 


\section{Introduction}

Dry weight estimation and nutrition assessment are vital procedures for hemodialysis (HD) patients because they affect the patient's prognosis and quality of life. Recently, bioimpedance analysis (BIA) has been widely used to analyze the volume, body composition, and nutritional status of dialysis patients [1-3]. BIA estimates body composition, including intracellular water (ICW), extracellular water (ECW), total body water (TBW), protein mass, mineral mass, skeletal muscle mass, and visceral fat area, based on measurements of impedance, reactance, and phase angle [4]. Segmental multi-frequency BIA has recently been developed and utilized in clinical practice, allowing measurement of not only the total body composition but also the segmental body composition $[5,6]$.

Although HD patients show periodic changes in fluid volume before and after dialysis, this does not directly indicate changes in specific BIA components. Significant increases in impedance, reactance, and phase angle after dialysis have been identified in a previous paper [7], but few studies have investigated detailed changes in BIA components preand post-HD, especially in East Asian HD patients, and information on segmental changes in BIA components is scarce. Therefore, this study aims to 1) compare the hydration and nutritional status of HD subjects with those of the general population using BIA, and 2) investigate the patterns of change in BIA component measurements in preand post-HD subjects, including segmental BIA values.

\section{Methods}

\section{Subjects and measurements}

This study was conducted with patients on maintenance HD (3 times a week) at two hospitals (Seoul National University Hospital and Seoul National University Boramae Medical Center) from July 2014 to August 2014. Only patients older than 18 who underwent BIA pre- and post-HD were enrolled. Patients suffering an acute illness within the previous 3 months and those with active malignancy, pulmonary edema, liver cirrhosis with ascites, New York Heart Association (NYHA) III or IV congestive heart failure, amputation, or lymphedema of the limbs were excluded. Clinical information, including past medical history, the cause of end-stage renal disease (ESRD), and HD vintage, was collected from the patient's medical chart. BIA was measured at mid-week dialysis sessions. Patients were instructed to take their meals 2 hours before dialysis to avoid interference effects of the meal, and were not allowed to eat during the course of dialysis. Once they were admitted to the dialysis unit, the patients changed into a hospital gown and were instructed to lie straight on the bed after measurement of their pre-HD weight. The patients rested for 10 minutes in a lying position and then BIA was measured using an S10 device (InBody, Seoul, Korea). Electrodes were attached to both hands and legs. The hand electrodes were attached to the thumb and the middle finger, while the foot electrodes were attached inside the medial side and outside the lateral side. After completion of pre-HD BIA measurements, blood tests were conducted simultaneously through venipuncture, and then dialysis was performed in the lying position. Once dialysis was completed, patients' post-HD weight was measured and BIA was measured in the same supine position. A chest X-ray was obtained before HD at the same day that BIA was conducted. Pretibial pitting edema was assessed by one trained physician before HD. The definition of leg edema was as follows: grade $1, \leq 2 \mathrm{~mm}$, disappears in 3 seconds; grade 2, 2 to $4 \mathrm{~mm}$, disappears in 10 to 15 seconds; grade 3,4 to $6 \mathrm{~mm}$, lasting > 1 minute; grade 4,6 to $8 \mathrm{~mm}$, lasting as long as 2 to 5 minutes.

For the general population-based control group, data on demographic factors, chest X-ray, and blood tests were recorded for health screening subjects at Seoul National University Hospital Healthcare System Gangnam Center on the same day that BIA was conducted, in July and August of 2014 and July and August of 2015. These measurements were obtained during the same period of the year to eliminate seasonal influences. Height, weight, and blood pressure (BP) were measured on the day of BIA assessment with the patients dressed in examination gowns and fasting. BIA was measured using a S720 device (InBody) in an upright position. Diabetes and hypertension were assessed based on replies to a standardized questionnaire, and those who responded that they had diabetes or hypertension were excluded. Subjects with a fasting glucose level $\geq 126 \mathrm{mg} / \mathrm{dL}$ or a hemoglobin Alc level $\geq 6.5 \%$ were considered to have diabetes, while those with a systolic BP of $>140 \mathrm{mmHg}$ or diastolic BP of $>90 \mathrm{mmHg}$ were considered to have hypertension. Subjects with high-sensitivity C-reactive protein level 
Kim, et al. Bioimpedance of hemodialysis patients

higher than $0.5 \mathrm{mg} / \mathrm{dL}$, cytopenia (white blood cell [WBC] count, $<4,000 / \mathrm{mm}^{3}$; hemoglobin, $<13 \mathrm{~g} / \mathrm{dL}$ for men, $<12$ $\mathrm{g} / \mathrm{dL}$ for women; platelet count, $<130,000 / \mathrm{mm}^{3}$ ) in their complete blood cell count, and serum creatinine levels higher than $1.1 \mathrm{mg} / \mathrm{dL}$ for females or $1.2 \mathrm{mg} / \mathrm{dL}$ for males were also excluded. Subjects with other abnormal findings were excluded at the discretion of the investigator.

The BIA components of ICW, ECW, TBW, ECW/TBW, lean tissue index (LTI), protein mass, mineral mass, fat mass, percent body fat (PBF), waist-hip ratio (WHR), visceral fat area (VFA), basal metabolic rate (BMR), soft lean mass, body cell mass (BCM), and phase angle were estimated through BIA measurements. Segmental TBW, segmental lean mass, segmental ECW/TBW, and segmental phase angle were estimated through segmental BIA in the HD group. The LTI, a marker of nutrition, was defined as the quotient of fat-free mass divided by height squared $\left(\mathrm{kg} / \mathrm{m}^{2}\right)$, BMR $(\mathrm{kcal})$ was calculated as fat-free mass $\times 21.6$ +370 , the estimated normal hydrated weight was calculated as the ideal weight when the ECW/TBW was 0.385 [8], and relative overhydration (\%) was defined as overhydration (L)/ECW (L) $\times 100$. Measured ultrafiltration (UF) volume difference was defined as the value obtained by subtracting the post-HD weight from the pre-HD weight, while predicted UF volume difference was defined as the difference in overhydration predicted by BIA based on pre- and post-HD ECW/TBW. In other words, measured UF volume difference designated the actual weight difference before and after HD, while predicted UF volume difference designated the weight difference before and after HD that was predicted by the BIA device. The average values of pre- and post-HD BIA were used when comparing the BIA of HD subjects with that of the control group.

All HD patients provided informed consent before their BIA and laboratory data were measured. However, since this study was a retrospective analysis and did not involve further invasive intervention, treatments, or costs to subjects, it was exempted from the requirement for consent from the control group. The study design was approved by the Institutional Review Board of Seoul National University Hospital, Seoul National University Boramae Hospital and Seoul National University Hospital Healthcare System Gangnam Center (H-1503-158-663). The participants' records were de-identified and analyzed anonymously. This study was performed in accordance with the Declaration of Helsinki.

\section{Extraction of controls using propensity score matching}

To ensure that controls had demographic characteristics similar to those of HD subjects, propensity score matching (PSM) was applied for age, sex, and height in health screening subjects without hypertension, diabetes, chronic kidney disease, or other comorbidities that could be detected from their medical history and laboratory or chest X-ray findings. Among a total of 5,976 subjects at the Seoul National University Hospital Healthcare System Gangnam Center, 298 were finally identified through PSM. The logit probability was estimated through logistic regression by setting either HD or control as a dependent variable and sex, age, and height as covariates. A propensity score was derived from the estimated logit probability value, and as a result $\mathrm{HD}$ and control groups were generated that were homogenous in terms of sex, age, and height.

In this study, the delta value for the expected probability was a logit score designating whether HD or control was defined as \pm 0.00005 , and then whether HD or control was extracted homogeneously at the aspects of sex, age, and height, using the matching method (Supplementary Table 1).

\section{Statistical analysis}

To determine the control group for the study, PSM was conducted with SAS version 9.3 (SAS Institute Inc., Cary, NC, USA). To test the significance of the difference between the time-average observed value of the HD group and the observed value of the control group, the independent two-sample $t$ test was conducted. Additionally, to test the difference between pre- and post-HD groups, the paired two-sample $t$ test was performed. To confirm the concordance of the measured and the predicted UF fluid volumes, the Bland-Altman test was performed. A backward stepwise regression model was used to search for the clinical markers most closely correlated with LTI. The data were analyzed with SPSS version 23.0 (IBM Corp., Armonk, NY, USA). All reported $P$ values are twotailed, and the statistical significance threshold was set at $P<0.05$. 


\section{Results}

Demographic characteristics and BIA components of the control and HD groups

After PSM, the distribution of sex, age, and height (sex [male], $54.4 \%$ vs. $53.1 \%$; age, 62 years vs. 63 years; height, $163 \mathrm{~cm}$ vs. $162 \mathrm{~cm}$ ) was similar between the control group $(\mathrm{n}=298)$ and the HD group $(\mathrm{n}=147)$. The prevalence of diabetes was $48 \%$ in the HD group whereas there were no cases of diabetes in the control group. In the HD group, the dialysis vintage was about 5 years, and the mean UF

Table 1. Clinical and laboratory information for the control and HD groups

\begin{tabular}{|c|c|c|c|}
\hline Variable & Control $(n=298)$ & $\mathrm{HD}(\mathrm{n}=147)$ & $P$ value \\
\hline Sex (male) & $162(54.4)$ & $78(53.1)$ & \\
\hline Age (yr) & $62.2 \pm 12.8$ & $63.1 \pm 13.6$ & \\
\hline Height (cm) & $163 \pm 8$ & $162 \pm 9$ & \\
\hline Diabetes & $0(0)$ & 70 (47.6) & \\
\hline Vintage (yr) & - & $4.86 \pm 3.25$ & \\
\hline UF volume (kg) & - & $1.89 \pm 1.14$ & \\
\hline spKt/V & - & $1.52 \pm 0.29$ & \\
\hline URR (\%) & - & $72.02 \pm 6.44$ & \\
\hline Pre SBP (mmHg) & $115 \pm 13$ & $139 \pm 22$ & $<0.001$ \\
\hline Pre DBP (mmHg) & $76 \pm 10$ & $73 \pm 14$ & 0.001 \\
\hline Post SBP (mmHg) & $115 \pm 13$ & $136 \pm 24$ & $<0.001$ \\
\hline Post DBP (mmHg) & $76 \pm 10$ & $74 \pm 13$ & 0.012 \\
\hline Pitting edema & - & $80(54.4)$ & \\
\hline CT ratio & $0.43 \pm 0.38$ & $0.52 \pm 0.07$ & $<0.001$ \\
\hline Hemoglobin (g/dL) & $14.3 \pm 1.5$ & $10.1 \pm 2.0$ & $<0.001$ \\
\hline WBC $\left(\times 10^{3} / \mathrm{mm}^{3}\right)$ & $5.22 \pm 1.45$ & $5.72 \pm 2.11$ & $<0.001$ \\
\hline Platelet $\left(\times 10^{3} / \mathrm{mm}^{3}\right)$ & $233 \pm 58$ & $181 \pm 85$ & $<0.001$ \\
\hline Protein $(\mathrm{g} / \mathrm{dL})$ & $7.22 \pm 0.44$ & $6.41 \pm 1.21$ & $<0.001$ \\
\hline Albumin (g/dL) & $4.53 \pm 0.22$ & $3.68 \pm 0.72$ & $<0.001$ \\
\hline AST (IU/L) & $23.6 \pm 8.3$ & $18.3 \pm 12.6$ & 0.019 \\
\hline ALT (IU/L) & $22.3 \pm 11.4$ & $14.7 \pm 10.6$ & 0.039 \\
\hline ALP (IU/L) & $54.2 \pm 15.1$ & $78.9 \pm 39.1$ & $<0.001$ \\
\hline BUN (mg/dL) & $15.0 \pm 3.3$ & $55.8 \pm 19.2$ & $<0.001$ \\
\hline Creatinine (mg/dL) & $0.83 \pm 0.17$ & $9.21 \pm 3.21$ & $<0.001$ \\
\hline Uric acid (mg/dL) & $5.47 \pm 1.34$ & $5.99 \pm 1.87$ & 0.056 \\
\hline Sodium (mmol/L) & $141.7 \pm 1.7$ & $133.5 \pm 22.9$ & $<0.001$ \\
\hline Potassium (mmol/L) & $4.27 \pm 0.33$ & $4.93 \pm 1.15$ & $<0.001$ \\
\hline Phosphorus (mg/dL) & $3.49 \pm 0.50$ & $4.54 \pm 1.65$ & $<0.001$ \\
\hline Calcium (mg/dL) & $9.18 \pm 0.30$ & $8.53 \pm 1.59$ & $<0.001$ \\
\hline Calcium $\times$ phosphorus & $32.1 \pm 5.1$ & $39.9 \pm 15.1$ & $<0.001$ \\
\hline Total cholesterol (mg/dL) & $198.9 \pm 34.7$ & $145.7 \pm 47.5$ & 0.029 \\
\hline Triglycerides (mg/dL) & $111.5 \pm 64.9$ & $100.8 \pm 84.5$ & 0.042 \\
\hline HbA1c (\%) & $5.51 \pm 0.22$ & $5.67 \pm 13.80$ & $<0.001$ \\
\hline $\mathrm{TIBC}(\mu \mathrm{g} / \mathrm{dL})$ & - & $218.9 \pm 65.5$ & \\
\hline Ferritin (ng/mL) & - & $164.4 \pm 237.2$ & \\
\hline PTH (pg/mL) & - & $128.4 \pm 169.6$ & \\
\hline
\end{tabular}

Data are presented as number (\%) or mean \pm standard deviation.

ALP, alkaline phosphatase; ALT, alanine aminotransferase; AST, aspartate aminotransferase; BUN, blood urea nitrogen; CT, chest thoracic; DBP, diastolic blood pressure; HbA1c, hemoglobin A1c; HD, hemodialysis; PTH, parathyroid hormone; SBP, systolic blood pressure; spKt/V, single-pool Kt/V; TIBC, transferrin iron binding capacity; UF, ultrafiltration; URR, urea reduction rate; WBC, white blood cell. 
volume was $1.89 \pm 1.14 \mathrm{~kg}$. The single-pool Kt/V (spKt/ $\mathrm{V}$ ) was 1.52 , and the urea reduction ratio (URR) was 72 , showing that HD was performed appropriately.

Compared to the control group, the systolic BP was higher and the diastolic BP was lower in the HD group. The mean chest thoracic (CT) ratio and WBC count of the HD group were higher, and the hemoglobin and platelet counts were lower. The nutritional indicators of lipid, protein, and albumin levels, aspartate aminotransferase (AST), and alanine aminotransferase (ALT) were lower in the HD group. The uric acid level in the HD group was higher, and the two groups also showed a difference in electrolytes (Table 1).

The average ECW/TBW and WHR were higher in the HD group; however, the other variables, including ICW, TBW, LTI, protein mass, mineral mass, BMR, fat, and PBF, were higher in the control group. Additionally, all components of BIA showed a difference between pre- and postHD. Body water, including ICW and ECW, decreased after HD, and the LTI, protein mass, mineral mass, and BMR accordingly decreased. Fat mass and PBF significantly increased after HD (Table 2).

Regarding the components associated with relative overhydration of the HD patients, the CT ratio, leg edema, AST, alkaline phosphatase, and sodium were found to be positively correlated with relative overhydration; among these, the positive correlation with the CT ratio $(r=0.425$,
$P<0.01$ ) was the strongest (Supplementary Table 2). In addition, relative overhydration was negatively correlated with the WBC count, platelet count, protein, albumin, blood urea nitrogen, creatinine, uric acid, potassium, phosphorus, triglycerides, and transferrin iron binding capacity (TIBC), and the negative correlation with creatinine $(r=-0.452, P<0.01)$ was the strongest (Supplementary Table 2). As the level of edema increased, mean relative overhydration increased ( $P$ for linear-by-linear association < 0.001) (Supplementary Fig. 1).

The Pearson correlation coefficients were calculated to verify the associations between the laboratory markers and LTI for the average values of the HD and control groups (Table 3). In the HD group, diastolic BP, creatinine, phosphorus, and TIBC showed positive correlations with LTI, while spKt/V, URR, and ferritin showed negative correlations. In the control group, systolic BP, diastolic $\mathrm{BP}$, hemoglobin, WBC, ALT, creatinine, uric acid, potassium, and triglyceride showed positive correlations with the LTI, whereas platelet count, sodium, phosphorus, and total cholesterol showed negative correlations. We further performed multivariate analysis using backward stepwise regression with the values that were significant $(P<0.05)$ in the univariate analysis. Among all subjects, females had a lower LTI. Using the average values from the HD subjects, lower URR and higher creatinine were closely correlated with a higher LTI. Among the controls,

Table 2. Comparison of BIA components in the control group with average (Avg.) values in the HD group and changes in the BIA components between pre- and post-HD

\begin{tabular}{|c|c|c|c|c|c|c|}
\hline Variable & Control $(n=298)$ & Pre-HD $(n=147)$ & Post-HD $(n=147)$ & Avg. $\mathrm{HD}(\mathrm{n}=147)$ & $P$ value $^{a}$ & $P$ value $^{b}$ \\
\hline BMI $\left(\mathrm{kg} / \mathrm{m}^{2}\right)$ & $22.88 \pm 2.68$ & $23.06 \pm 3.63$ & $22.33 \pm 3.51$ & $22.69 \pm 3.56$ & $<0.001$ & 0.002 \\
\hline ICW (L) & $21.62 \pm 4.44$ & $19.84 \pm 4.12$ & $19.06 \pm 4.10$ & $19.45 \pm 4.10$ & 0.001 & 0.002 \\
\hline ECW (L) & $13.12 \pm 2.51$ & $13.40 \pm 2.69$ & $12.49 \pm 2.62$ & $12.95 \pm 2.64$ & $<0.001$ & 0.744 \\
\hline TBW (L) & $34.73 \pm 6.91$ & $33.24 \pm 6.76$ & $31.55 \pm 6.65$ & $32.40 \pm 6.69$ & $<0.001$ & 0.033 \\
\hline ECW/TBW & $0.38 \pm 0.01$ & $0.40 \pm 0.01$ & $0.40 \pm 0.01$ & $0.40 \pm 0.01$ & $<0.001$ & $<0.001$ \\
\hline LTI $\left(\mathrm{kg} / \mathrm{m}^{2}\right)$ & $16.80 \pm 2.03$ & $16.98 \pm 1.96$ & $16.14 \pm 1.95$ & $16.56 \pm 1.94$ & $<0.001$ & 0.035 \\
\hline Protein (kg) & $9.31 \pm 1.91$ & $8.58 \pm 1.79$ & $8.24 \pm 1.77$ & $8.41 \pm 1.78$ & $<0.001$ & 0.002 \\
\hline Mineral (kg) & $3.19 \pm 0.59$ & $3.05 \pm 0.56$ & $2.90 \pm 0.55$ & $2.97 \pm 0.56$ & $<0.001$ & 0.039 \\
\hline BMR (kcal) & $1,390 \pm 203$ & $1,339 \pm 196$ & $1,292 \pm 193$ & $1,316 \pm 194$ & $<0.001$ & 0.018 \\
\hline Fat (kg) & $16.83 \pm 4.64$ & $15.69 \pm 7.76$ & $15.98 \pm 7.89$ & $15.83 \pm 7.81$ & $<0.001$ & $<0.001$ \\
\hline PBF (\%) & $26.38 \pm 5.95$ & $25.32 \pm 9.89$ & $26.65 \pm 10.45$ & $25.99 \pm 10.14$ & $<0.001$ & $<0.001$ \\
\hline WHR & $0.88 \pm 0.05$ & $0.88 \pm 0.09$ & $0.91 \pm 0.09$ & $0.90 \pm 0.09$ & $<0.001$ & $<0.001$ \\
\hline
\end{tabular}

Data are presented as mean \pm standard deviation.

BIA, bioimpedance analysis; BMI, body mass index; BMR, basal metabolic rate; ECW, extracellular water; HD, hemodialysis; ICW, intracellular water; LTI, lean tissue index; PBF, percent body fat; TBW, total body water; WHR, waist-hip ratio.

${ }^{\mathrm{a}} P$ value pre-HD compared with post $-\mathrm{HD}$, and ${ }^{\mathrm{b}} P$ value of the control group compared with Avg. HD. 
Table 3. Analysis of LTI and laboratory markers of nutrition in the control group and the average (Avg.) HD group: univariate correlation

\begin{tabular}{|c|c|c|c|c|}
\hline \multirow{2}{*}{ Variable } & \multicolumn{2}{|c|}{ LTI (Avg. HD) } & \multicolumn{2}{|c|}{ LTI (control) } \\
\hline & $r$ & $P$ & $r$ & $P$ \\
\hline SBP & 0.159 & 0.059 & 0.225 & $<0.001$ \\
\hline DBP & 0.212 & 0.012 & 0.389 & $<0.001$ \\
\hline spKt/V & -0.446 & $<0.001$ & & \\
\hline URR & -0.474 & $<0.001$ & & \\
\hline Hemoglobin & 0.005 & 0.955 & 0.571 & $<0.001$ \\
\hline WBC & 0.029 & 0.735 & 0.198 & 0.001 \\
\hline Platelet & 0.059 & 0.486 & -0.159 & 0.006 \\
\hline Protein & 0.037 & 0.660 & -0.148 & 0.010 \\
\hline Albumin & 0.036 & 0.666 & -0.008 & 0.884 \\
\hline AST & 0.001 & 0.988 & 0.070 & 0.231 \\
\hline ALT & 0.131 & 0.118 & 0.280 & $<0.001$ \\
\hline ALP & -0.079 & 0.349 & -0.011 & 0.847 \\
\hline BUN & 0.068 & 0.422 & 0.093 & 0.110 \\
\hline Creatinine & 0.310 & $<0.001$ & 0.698 & $<0.001$ \\
\hline Uric acid & -0.130 & 0.123 & 0.517 & $<0.001$ \\
\hline Sodium & 0.040 & 0.639 & -0.238 & $<0.001$ \\
\hline Potassium & 0.159 & 0.057 & 0.138 & 0.017 \\
\hline Phosphorus & 0.192 & 0.022 & -0.462 & $<0.001$ \\
\hline Calcium & -0.024 & 0.775 & -0.112 & 0.054 \\
\hline Total cholesterol & -0.065 & 0.443 & -0.280 & $<0.001$ \\
\hline Triglycerides & 0.034 & 0.691 & 0.250 & $<0.001$ \\
\hline TIBC & 0.171 & 0.043 & & \\
\hline Ferritin & -0.239 & 0.004 & & \\
\hline
\end{tabular}

ALP, alkaline phosphatase; ALT, alanine aminotransferase; AST, aspartate aminotransferase; BUN, blood urea nitrogen; DBP, diastolic blood pressure; HD, hemodialysis; LTI, lean tissue index; SBP, systolic blood pressure; spKt, singlepool Kt; TIBC, transferrin iron binding capacity; URR, urea reduction rate; WBC, white blood cell. . higher diastolic BP and creatinine were correlated with a higher LTI (Table 4).

\section{Pre/Post-HD changes in BIA components}

For body water distribution, the rate of change per $1 \mathrm{~kg}$ body weight after HD (\%/kg) was higher for ECW than for ICW (ECW vs. ICW, 4.26 vs. $1.99, P<0.001$ by the paired $t$ test). ICW $(1.99 \% / \mathrm{kg})$, TBW $(2.86 \% / \mathrm{kg})$, estimated protein mass $(2.29 \% / \mathrm{kg})$, mineral mass $(2.43 \% / \mathrm{kg})$, and soft lean mass $(2.74 \% / \mathrm{kg})$ were higher pre-HD than post-HD, and protein or mineral mass was estimated to be higher when patients were more overhydrated (pre-HD vs. postHD: protein [kg], 8.58 vs. 8.24; mineral [kg], 3.05 vs. 2.90). The nutritional indices, such as BCM $(2.02 \% / \mathrm{kg})$ or LTI $(2.73 \% / \mathrm{kg})$, were also higher in the pre-HD than in the post-HD (Supplementary Table 3), whereas fat $(-1.79 \%$ / $\mathrm{kg}$ ) and WHR $(-3.28 \% / \mathrm{kg})$ decreased. In addition, the estimated normal hydrated weight that was estimated by ECW/TBW before and after HD was predicted to be approximately $1.5 \mathrm{~kg}$ higher pre-HD than post-HD.

The scatter plot of the predicted UF volume difference versus the measured UF volume difference before and after HD shows a high correlation $(r=0.924, P<0.001)$ except for several outliers (Fig. 1). The mean \pm standard deviation of the actual UF volume difference measured in all HD subjects was $1.89 \pm 1.14 \mathrm{~kg}$, while that of the predicted UF volume difference was $1.46 \pm 1.02 \mathrm{~kg}$. The correlation between the estimated and measured UF seemed to be very strong (Fig. 1), with a quite modest bias of $<0.5$ $\mathrm{kg}$, although the two values were not the same statisti-

Table 4. Analysis of lean tissue index and laboratory markers of nutrition in the control group and the average HD group: multivariate analysis using backward stepwise regression

\begin{tabular}{|c|c|c|c|c|c|}
\hline & $\mathrm{B}$ & SE & $\beta$ & $\mathrm{t}$ & $P$ value \\
\hline \multicolumn{6}{|l|}{ Average HD } \\
\hline Constant & 24.068 & 1.954 & - & 12.319 & $<0.001$ \\
\hline Age (yr) & -0.019 & 0.01 & -0.136 & -1.854 & 0.066 \\
\hline Female & -1.063 & 0.287 & -0.274 & -3.707 & $<0.001$ \\
\hline $\mathrm{DBP}(\mathrm{mmHg})$ & 0.017 & 0.01 & 0.114 & 1.666 & 0.098 \\
\hline URR (\%) & -0.115 & 0.022 & -0.375 & -5.161 & $<0.001$ \\
\hline Creatinine (mg/dL) & 0.131 & 0.052 & 0.189 & 2.508 & 0.013 \\
\hline \multicolumn{6}{|l|}{ Control } \\
\hline Constant & 15.363 & 0.817 & - & 18.805 & $<0.001$ \\
\hline Female & -2.382 & 0.205 & -0.586 & -11.636 & $<0.001$ \\
\hline $\mathrm{DBP}(\mathrm{mmHg})$ & 0.019 & 0.007 & 0.096 & 2.761 & 0.006 \\
\hline Creatinine (mg/dL) & 2.757 & 0.576 & 0.225 & 4.789 & $<0.001$ \\
\hline
\end{tabular}

DBP, diastolic blood pressure; HD, hemodialysis; SE, standard error; URR, urea reduction rate. 
A

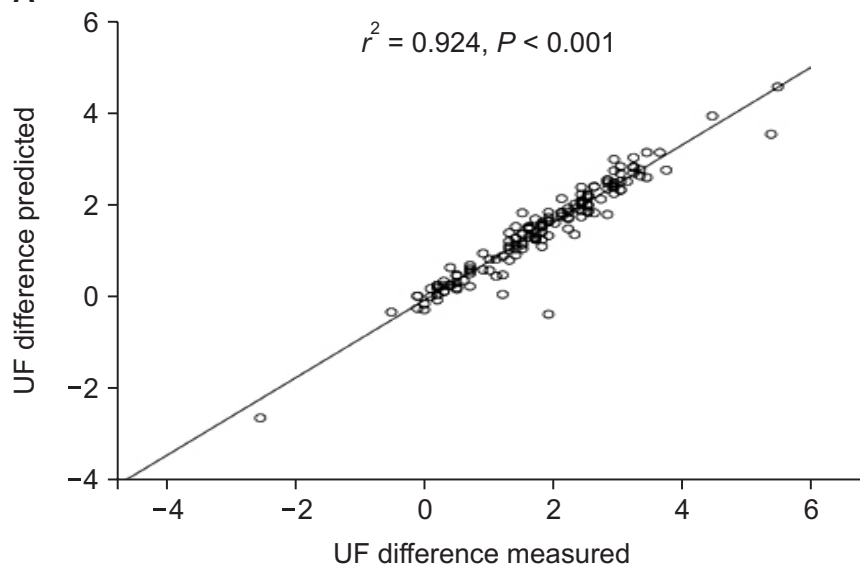

B

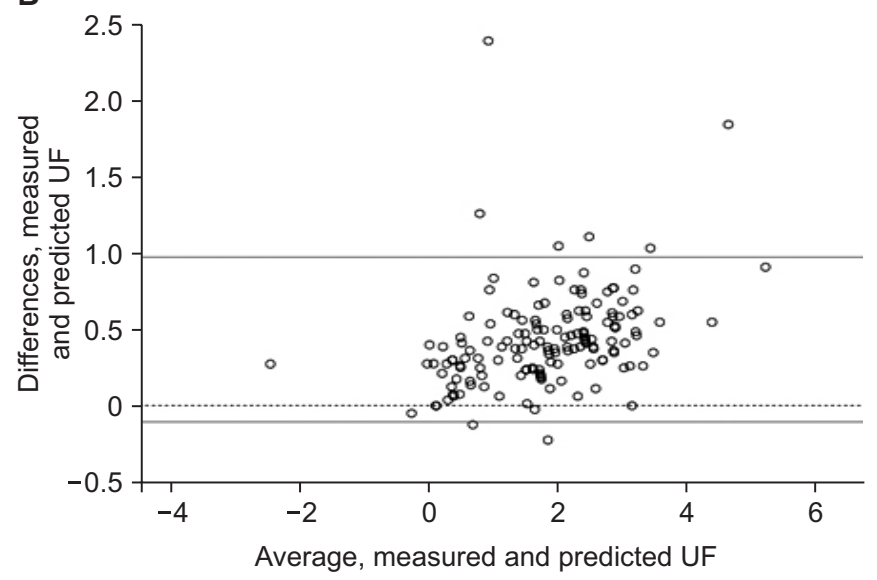

Figure 1. Correlation and Bland-Altman plot for the agreement between measured and estimated ultrafiltration (UF) volume. (A) Correlation between measured and estimated ultrafiltration (UF) volume. (B) Bland-Altman plot for the agreement between measured and estimated UF volume.

Table 5. Segmental changes in bioimpedance analysis between pre- and post-HD $(n=147)$

\begin{tabular}{|c|c|c|c|c|c|}
\hline Variable & Pre-HD & Post-HD & $\mathrm{A}(\%)$ & $\mathrm{B}(\% / \mathrm{kg})$ & $T$ \\
\hline \multicolumn{6}{|l|}{ Segmental water } \\
\hline Arm without VA & $1.868 \pm 0.527$ & $1.753 \pm 0.532$ & 6.17 & 2.87 & 11.445 \\
\hline Arm with VA & $1.941 \pm 0.579$ & $1.865 \pm 0.610$ & 3.90 & 0.77 & 6.071 \\
\hline Trunk & $15.808 \pm 3.234$ & $15.345 \pm 3.249$ & 2.93 & 0.96 & 10.398 \\
\hline Right leg & $5.299 \pm 1.427$ & $4.922 \pm 1.330$ & 7.13 & 5.78 & 22.844 \\
\hline Left leg & $5.257 \pm 1.401$ & $4.901 \pm 1.317$ & 6.77 & 5.34 & 24.772 \\
\hline \multicolumn{6}{|l|}{ Segmental lean } \\
\hline Arm without VA & $2.391 \pm 0.673$ & $2.247 \pm 0.680$ & 6.04 & 2.82 & 11.382 \\
\hline Arm with VA & $2.480 \pm 0.736$ & $2.386 \pm 0.776$ & 3.81 & 0.69 & 6.049 \\
\hline Trunk & $20.149 \pm 4.147$ & $19.608 \pm 4.168$ & 2.69 & 0.65 & 9.699 \\
\hline Right leg & $6.743 \pm 1.818$ & $6.280 \pm 1.700$ & 6.87 & 5.52 & 22.481 \\
\hline Left leg & $6.685 \pm 1.785$ & $6.251 \pm 1.683$ & 6.48 & 5.03 & 24.480 \\
\hline \multicolumn{6}{|l|}{ ECW/TBW } \\
\hline Arm without VA & $0.389 \pm 0.008$ & $0.385 \pm 0.009$ & 1.15 & 0.61 & 14.349 \\
\hline Arm with VA & $0.394 \pm 0.008$ & $0.391 \pm 0.010$ & 0.88 & 0.29 & 8.935 \\
\hline Trunk & $0.403 \pm 0.012$ & $0.396 \pm 0.015$ & 1.72 & 1.37 & 22.107 \\
\hline Right leg & $0.408 \pm 0.015$ & $0.400 \pm 0.017$ & 1.98 & 1.70 & 19.317 \\
\hline Left leg & $0.409 \pm 0.014$ & $0.401 \pm 0.016$ & 2.11 & 1.78 & 21.972 \\
\hline \multicolumn{6}{|l|}{ Phase angle } \\
\hline Arm without VA & $4.270 \pm 0.917$ & $4.629 \pm 1.038$ & -8.42 & -5.33 & -12.050 \\
\hline Arm with VA & $3.854 \pm 0.820$ & $4.100 \pm 0.945$ & -6.38 & -2.37 & -7.349 \\
\hline Trunk & $4.927 \pm 1.086$ & $5.243 \pm 1.211$ & -6.41 & -3.50 & -5.259 \\
\hline Right leg & $4.027 \pm 1.216$ & $4.586 \pm 1.409$ & -13.88 & -12.84 & -16.777 \\
\hline Left leg & $3.915 \pm 1.177$ & $4.447 \pm 1.383$ & -13.59 & -11.74 & -15.813 \\
\hline
\end{tabular}

Data are presented as mean \pm standard deviation.

ECW, extracellular water; HD, hemodialysis; TBW, total body water; VA, vascular access.

$A=($ pre - post $) /$ pre (unit, \%), $B=[($ pre - post $) /$ pre $] /$ pre weight - post weight $)($ unit, \%/kg).

$P$ values are for comparisons between pre-HD and post-HD values; the $P$ value of every variable was $<0.001$. 
cally.

To verify the significance of the segmental changes in BIA between pre- and post-HD, the paired-samples $t$ test was performed (Table 5). The pre/post changes were statistically significant in all areas of segmental water, segmental lean mass, ECW/TBW, and phase angle. For segmental water, ECW/TBW, and segmental lean mass, the post-HD means decreased compared with pre-HD, whereas for the phase angle the post-HD mean increased compared with pre-HD.

Excluding the arms with vascular access, the mean segmental water values in the extremities changed by $0.96 \% / \mathrm{kg}$ to $5.78 \% / \mathrm{kg}$, the segmental lean mass changed by $0.65 \% / \mathrm{kg}$ to $5.52 \% / \mathrm{kg}$, and the ECW/TBW changed by $1.15 \% / \mathrm{kg}$ to $2.11 \% / \mathrm{kg}$. The mean phase angle changed by approximately $5.33 \%$ at the arm with vascular access, $3.50 \%$ at the trunk, and $11.74 \%$ and $12.84 \%$ at the lower limbs, revealing that the phase angle showed the greatest proportional change.

\section{Discussion}

To understand the features of the BIA components in HD subjects, PSM was performed for age, sex, and height in health screening subjects without hypertension, diabetes, or chronic kidney disease. After PSM, the difference in the BIA components between the two groups was determined and the patterns of change in the BIA components pre-/post-HD were investigated, including the segmental components. HD patients had more ECW than the normal population; additionally, most nutritional indices were lower and the VFA was slightly higher in HD subjects. The relative overhydration of HD patients showed the highest correlation with the CT ratio and moderate correlation with leg edema. In contrast to the control group, the LTI of HD patients was not correlated with nutritional markers. In this study, we found that pre- and post-HD values of ECW/TBW were not identical but correlated very well; therefore, the estimated normohydrated weight using ECW/TBW can be a good marker for determining dry weight. In terms of segmental BIA changes, the greatest change occurred in the leg and the phase angle showed the greatest proportional changes.

In our study we adopted average BIA parameters as volume and nutritional markers of HD. This was because HD subjects experience cyclic volume changes, making it more appropriate to use the average volume status from pre- and post-HD data rather than the post-HD BIA parameters. Based on the study of Papakrivopoulou et al [9], we compared the average values of HD patients to those of controls because of periodic volume change fluctuations before and after dialysis in HD patients.

In the present study, the second BIA measurements were made 10 minutes after HD based on the protocol of Di Iorio et al [3]. These authors identified changes in impedance, reactance, and phase angle pre- and post$\mathrm{HD}$, and showed that the values measured post-HD were maintained for 2 hours after HD. Thereafter, the values continuously increased until the next round of HD. Therefore, this study was conducted based on the prediction that body composition indices would be constant from immediately after HD to 2 hours after HD, and then begin cyclical changes starting at 2 hours after HD. As HD patients experience overhydration until the subsequent $\mathrm{HD}$, it is reasonable to evaluate overhydration using the mean pre- and post-HD values.

BIA has been widely used in previous studies as a helpful tool for estimating dry weight and body composition in HD subjects $[2,10]$. Since it is highly reproducible, BIA has the advantage of low interobserver error [11]. In addition, correlations of BIA with dual X-ray absorption, which is the gold standard for body composition measurement, and subjective global assessment, which is the gold standard for nutritional indices, have been identified in HD subjects [12,13]. As most indices provided by BIA have components estimated by the impedance associated with the amount of body water [14], pre-/post$\mathrm{HD}$ changes in the above indices are expected and must be considered when interpreting the BIA results of HD subjects. The change in each index needs to be examined before HD, when the water is most excessive, and after HD, which reflects the patient's dry weight status, but these indices have not yet been precisely investigated to date. Chua et al [1] argued that nutritional assessments using lean body mass and BCM measurements were significantly confounded by hydration status, and fat mass was found to be relatively constant using whole-body BIA in a small number of HD subjects. This result is also consistent with our analysis. However, the study of Chua et al [1] had limitations in that it used whole-body BIA rather than segmental BIA and included incident HD subjects drawn from hospitalized patients with ESRD whose hy- 
dration status was not stable. In addition, they investigated the agreement of measured UF with TBW and ECW, and the degree of precision was poor when compared to the agreement between acute weight change and UF. In our study we showed a similar relationship, but it was confirmed that the simple terms of predicted and measured normal hydrated weight did not match and a systematic error in BIA was also found. Panorchan et al [6] measured segmental BIA in 676 European subjects and reported the trend that skeletal muscle mass increases and PBF decreases when overhydration is present. Our study showed a similar result to that of Panorchan et al [6], although the change in fat was not statistically significant. The reasons for this may include the small sample size of our study, such that statistical significance was not shown, or population differences between European subjects and the East Asian subjects from which our sample was drawn [1]. The excellent study of Panorchan et al [6] also compared pre- and post-HD BIA parameters. However, our study showed that the weight predicted using pre- and post-HD parameters was not the same, although the values were not significantly different, therefore we sought to compare more detailed parameters.

Although HD patients were overhydrated compared with the control group the nutritional indices were lower, which is consistent with the results of previous studies [15]. In the present study, overhydration itself increased nutritional components such as muscle mass, BCM, and phase angle. Considering that HD subjects were still overhydrated post-HD (ECW/TBW, 0.396), it might be predicted that they would be more malnourished than the measured BIA components indicated. In the present study, the average LTI of overall HD patients calculated using the mean ECW/TBW value of the control group (0.378) was lower than the value observed post-HD (LTI, predicted value when $\mathrm{ECW} / \mathrm{TBW}=0.378$; post-HD vs. average in HD patients, $16.12 \pm 1.95$ vs. $16.56 \pm 1.95, P<$ $0.001)$. Likewise, we can predict that the fat mass of the HD group calculated using an ECW/TBW value of 0.378 was far greater than that observed in the post-HD subjects. This means that for the same volume as the control group, HD patients would have a lower LTI and more fat. Conventional nutritional laboratory markers were not correlated in HD patients. Moreover, albumin was not a significant predictor in either group because it has limited value as a nutritional marker in HD subjects [16].
The distribution of albumin in HD subjects was generally in the 3 to $4 \mathrm{~g} / \mathrm{dL}$ range whereas most of the normal controls had albumin levels of $4 \mathrm{~g} / \mathrm{dL}$; we concluded that this difference had no discernible power.

BCM and LTI, used as nutritional indices [17], were higher in the pre-HD measurements than in the post-HD measurements (BCM, $2.02 \% / \mathrm{kg}$; LTI, $2.73 \% / \mathrm{kg}$ ). The normal hydrated weight at the pre-HD was also higher than that at the post-HD (normal hydrated weight, $1.22 \% / \mathrm{kg}$ ). Since both ECW and ICW decreased, the normal hydrated weight, which is estimated using ECW/TBW, seems to have changed.

We also examined the patterns of changes in segmental BIA. In terms of segmental water, the pre-/post-HD changes in the legs were proportionally higher than those in the arms. Segmental lean mass and ECW/TBW showed a similar pattern. The reason for this seems to be that subjects who walked upright had more severe edema in the legs than in the arms and torso before HD whereas the degree of edema in the arms and legs equalized in the supine position after HD. Phase angle increased by $5.3 \%$ / $\mathrm{kg}$ in arms without vascular access, $3.5 \% / \mathrm{kg}$ in the trunk, and $11.74 \% / \mathrm{kg}$ and $12.84 \% / \mathrm{kg}$ in the lower limbs after $\mathrm{HD}$, which were the proportionally greatest changes between pre- and post-HD among the segmental BIA components. Although phase angle is currently used as a nutritional marker in various populations $[18,19]$, it must be carefully interpreted when used as a nutritional marker in HD patients because this index dramatically changes in this population according to their level of overhydration.

The strengths of this study are as follows. First, the study protocol was designed to take into account seasonal and dietary influences in the maintenance HD subjects, thereby minimizing their effects on the measurements. As body water and body composition are affected by seasons, we synchronized the months in which BIA measurements were made in HD subjects and the control group to rule out seasonal influences. Moreover, the measurements were taken after fasting to rule out the effects of food intake. Second, a control group with homogeneous characteristics was derived using PSM, which adds weight to the observation that the principal differences between healthy subjects and dialysis patients are nutritional, causing relative overhydration. Finally, the correlations between BIA components and the CT ratio 
and leg edema, which are frequently used as bedside indicators, were examined. The study has the limitation that different BIA devices were used in the control and HD groups. Additionally, BIA of the control group was measured in the upright position, rather than in the supine position used for the HD group. However, both BIA devices were produced by the same company (Inbody), were analyzed at the same time, and were found to have the same BIA parameters [20]. Moreover, since the BIA values obtained using the S10 device in the supine position were corrected for the values obtained with the S720 device in a standing position, this is unlikely to have been a major problem. Finally, the lack of data on residual kidney function data is a weakness of the study, although this only applied to $8 \%$ of the subjects. We included subjects with any diseases, including diabetes or hypertension, in the control group. Choosing a diseasefree control group rather than a control group based on normal function might have exaggerated the findings of the study.

The primary purpose of this study was to compare BIA components between HD subjects and normal controls. HD subjects had more ECW compared with the general population; in addition, most nutritional indices were lower and visceral fat was slightly higher. In the control group known laboratory markers of nutrition were highly correlated with LTI, but this correlation was insignificant in HD subjects. Since most indices changed by a statistically significant degree according to the volume status, careful interpretation is necessary. It was almost possible to predict UF volume differences using BIA, but the increase in leg phase angle post-HD must be interpreted with caution.

\section{Conflicts of interest}

All authors have no conflicts of interest to declare.

\section{Acknowledgments}

This research was supported by a grant of National Research Foundation, Republic of Korea (grant number: NRF-2016R1D1A1B03934173). The authors greatly appreciate the dedicated efforts of Eun-song Kim and Eunjin Kang in the measurement of BIA components.

\section{References}

[1] Chua HR, Xiang L, Chow PY, et al. Quantifying acute changes in volume and nutritional status during haemodialysis using bioimpedance analysis. Nephrology (Carlton) 17:695702,2012

[2] Davies SJ, Davenport A. The role of bioimpedance and biomarkers in helping to aid clinical decision-making of volume assessments in dialysis patients. Kidney Int 86:489496, 2014

[3] Di Iorio BR, Scalfi L, Terracciano V, Bellizzi V. A systematic evaluation of bioelectrical impedance measurement after hemodialysis session. Kidney Int 65:2435-2440, 2004

[4] Broers NJ, Martens RJ, Cornelis T, et al. Body composition in dialysis patients: a functional assessment of bioimpedance using different prediction models. J Ren Nutr 25:121128, 2015

[5] Wahba IM, Bennett WM. Increased vascular resistance and not salt retention characterizes cyclosporine A-induced hypertension: report in an anuric patient. Am J Transplant 7:2042-2046, 2007

[6] Panorchan K, Nongnuch A, El-Kateb S, Goodlad C, Davenport A. Changes in muscle and fat mass with haemodialysis detected by multi-frequency bioelectrical impedance analysis. Eur J Clin Nutr 69:1109-1112, 2015

[7] Cunningham JJ. Body composition as a determinant of energy expenditure: a synthetic review and a proposed general prediction equation. Am J Clin Nutr 54:963-969, 1991

[8] Thanakitcharu P, Jirajan B. Early detection of subclinical edema in chronic kidney disease patients by bioelectrical impedance analysis. J Med Assoc Thai 97 Suppl 11:S1-S10, 2014

[9] Papakrivopoulou E, Booth J, Pinney J, Davenport A. Comparison of volume status in asymptomatic haemodialysis and peritoneal dialysis outpatients. Nephron Extra 2:48-54, 2012

[10] Castellano S, Palomares I, Molina M, et al. Clinical, analytical and bioimpedance characteristics of persistently overhydrated haemodialysis patients. Nefrologia 34:716-723, 2014

[11] Davenport A, Willicombe M. Comparison of fluid status in patients treated by different modalities of peritoneal dialysis using multi-frequency bioimpedance. Int J Artif Organs 32:779-786, 2009

[12] Furstenberg A, Davenport A. Comparison of multifrequency bioelectrical impedance analysis and dual-energy X-ray 
absorptiometry assessments in outpatient hemodialysis patients. Am J Kidney Dis 57:123-129, 2011

[13] Garagarza C, Joao-Matias P, Sousa-Guerreiro C, et al. Nutritional status and overhydration: can bioimpedance spectroscopy be useful in haemodialysis patients? Nefrologia 33:667-674, 2013

[14] Kotanko P, Levin NW, Zhu F. Current state of bioimpedance technologies in dialysis. Nephrol Dial Transplant 23:808812, 2008

[15] Plum J, Schoenicke G, Kleophas W, et al. Comparison of body fluid distribution between chronic haemodialysis and peritoneal dialysis patients as assessed by biophysical and biochemical methods. Nephrol Dial Transplant 16:23782385, 2001

[16] Ikizler TA. The use and misuse of serum albumin as a nutritional marker in kidney disease. Clin J Am Soc Nephrol
7:1375-1377, 2012

[17] Marcelli D, Usvyat LA, Kotanko P, et al. Body composition and survival in dialysis patients: results from an international cohort study. Clin J Am Soc Nephrol 10:1192-1200, 2015

[18] Fein PA, Gundumalla G, Jorden A, Matza B, Chattopadhyay J, Avram MM. Usefulness of bioelectrical impedance analysis in monitoring nutrition status and survival of peritoneal dialysis patients. Adv Perit Dial 18:195-199, 2002

[19] Genton L, Norman K, Spoerri A, et al. Bioimpedance-derived phase angle and mortality among older people. Rejuvenation Res 20:118-124, 2016

[20] Anderson LJ, Erceg DN, Schroeder ET. Utility of multi-frequency bioelectrical impedance compared to deuterium dilution for assessment of total body water. Nutr Diet 72:183-189, 2015 